\title{
Expansion of Linear Analysis of Rayleigh-Taylor Interface Instability of Metal Materials
}

\author{
Xinbo Bai' ${ }^{1}$, Tao Wang2, Yuxuan Zhu ${ }^{3}$, Guoqiang Luo ${ }^{3}$ \\ ${ }^{1}$ College of Physical Science and Technology, Sichuan University, Chengdu, China \\ ${ }^{2}$ Institute of Fluids Physics, China Academy of Engineering Physics, Mianyang, China \\ ${ }^{3}$ State Key Laboratory of Advanced Technology for Materials Synthesis and Processing, Wuhan University of Technology, \\ Wuhan, China \\ Email: ${ }^{\star}$ luoguoqiang1980@sina.com
}

How to cite this paper: Bai, X.B., Wang, T., Zhu, Y.X. and Luo, G.Q. (2018) Expansion of Linear Analysis of Rayleigh-Taylor Interface Instability of Metal Materials. World Journal of Mechanics, 8, 94-106. https://doi.org/10.4236/wjm.2018.84008

Received: February 19, 2018

Accepted: April 27, 2018

Published: April 30, 2018

Copyright () 2018 by authors and Scientific Research Publishing Inc. This work is licensed under the Creative Commons Attribution International License (CC BY 4.0).

http://creativecommons.org/licenses/by/4.0/

\section{(c) (i) Open Access}

\begin{abstract}
The linear analysis of the Rayleigh-Taylor instability in metal material is extended from the perfect plastic constitutive model to the Johnson-Cook and Steinberg-Guinan constitutive model, and from the constant loading to a time-dependent loading. The analysis is applied to two Rayleigh-Taylor instability experiments in aluminum and vanadium with peak pressures of $20 \mathrm{GPa}$ and $90 \mathrm{GPa}$, and strain rates of $6 \times 10^{6} \mathrm{~s}^{-1}$ and $3 \times 10^{7} \mathrm{~s}^{-1}$ respectively. When the time-dependent loading and the Steinberg-Guinan constitutive model are used in the linear analysis, the analytic results are in close agreement with experiments quantitatively, which indicates that the method in this paper is applicable to the Rayleigh-Taylor instability in aluminum and vanadium metal materials under high pressure and high strain rate. From these linear analyses, we find that the constitutive models and the loading process are of crucial importance in the linear analysis of the Rayleigh-Taylor instability in metal material, and a better understanding of the Rayleigh-Taylor instability in metals is gained. These results will serve as important references for evolving high-pressure, high-strain-rate experiments and numerical simulations.
\end{abstract}

\section{Keywords}

Linear Analysis, Rayleigh-Taylor Instability, Time-Dependent Loading, Constitutive Model

\section{Introduction}

The perturbed interface in a metal material will undergo growth and mixing in- 
duced by interfacial instability under strong shock, high acceleration, and shear driving. There are numerous research works [1] [2] [3] [4] [5] covering linear analysis and numerical simulation of the Rayleigh-Taylor instability (RTI) of gases and liquids. Researchers have gradually developed various experimental devices, such as the linear electric motor [6] and the shock wave tube [7] [8], and some diagnostic techniques such as particle-image velocimetry (PIV) [9] and planar laser-induced fluorescence (PLIF) [10]. For metals, the loading technique for the instability involves high pressure and high strain rate, so it is difficult to implement. Because a high-accuracy, high-resolution diagnostic technique for the RTI in metals has not yet been developed, high-level experimental work of the RTI in metals has been lacking. For numerical simulation, computation of the RTI in metals, involving the physical modeling of the material encompassing material physical behaviors, constitutive models, equations of state, and phase transformation dynamics, is also an interesting research topic.

The RTI in metals has been studied by many authors, since the work of Miles in 1968 [11]. These studies could be categorized into three groups: the first group is the method based on the energy-balanced equation which was used by Miles [11], Robinson and Swegle [12]; the second group is the method based on the Eulerian equations for a continuum medium which was used by Plohr and Sharp [13], Terrones [14]; the last one is the method recently proposed by Piriz et al. [15] [16] who derived the amplitude equation from the Newton second law. Although these methods presented similar features for the RTI in metals, they gave different quantitative results for the growth rate and the cutoff perturbation wave number. Although the first method and the third method should be physically equivalent, they showed different quantitative results. As shown in [16], "the instability is not controlled by the details of the behavior of a material point but rather it is governed by the bulk motion of the mass contained within the region with a thickness $k^{-1}$, we think the method based on the energy balance is more physically realistic.

In this paper, we derive the equation of the perturbation amplitude based on the energy balance. There are three improvements over the classical linear analysis of the RTI in metals presented in this work. First, because the constant pressure driving used in previous works differs from the actual experimental driving process and is not applicable for a quantitative analysis of experiments, we introduce time-dependent pressure driving into the linear analysis of the RTI in metals. Second, we perform a linear analysis of the RTI in metals based on the Johnson-Cook (JC) and Steinberg-Guinan (SG) constitutive models. However, in the previous published papers [11]-[16], the perfect plastic (PP) constitutive model is used for the linear analysis of the RTI in metals to obtain the analytic solution of the perturbation growth. When the constitutive models that better characterize the stress-strain relation in metals under dynamic loading, such as the JC and SG constitutive models, are used for the linear analysis of the RTI in metals, analytic solutions cannot be found, and a numerical solution is needed. 
Third, we validate the linear analysis of the RTI in metals experimentally in aluminum and vanadium under high-pressure, high-strain-rate driving, using two Omega laser experiments.

\section{Analytical Formulation of the Problem}

For the linear analysis of interface instability of a finite thickness plate (Figure 1 ), a velocity field is introduced with the result of Taylor's inviscid, impressible fluid

$$
u_{x}=\dot{\xi} \mathrm{e}^{-k y} \sin k x, \quad u_{y}=\dot{\xi} \mathrm{e}^{-k y} \cos k x .
$$

where $\xi(t)$ is the perturbation amplitude on the interface $(y=0), \quad k=2 \pi / \lambda$ is the wave number.

The average potential and kinetic energies within a wavelength in the accelerated reference frame that moves with the plate are, respectively

$$
\begin{gathered}
\langle V\rangle=-\frac{1}{4} \rho g\left(1-\mathrm{e}^{-2 k h}\right) \xi^{2}(t) \\
\langle T\rangle=\frac{1}{4} \frac{\rho}{k}\left(1-\mathrm{e}^{-2 k h}\right) \dot{\xi}^{2}(t)
\end{gathered}
$$

where $\rho$ is the material density, $g=P / \rho h$ is the acceleration of the plate, $P$ is the driving pressure, $h$ is the thickness of the plate. In a typical planar Rayleigh-Taylor experiment, the driving pressure is generated by a flowing plasma atmosphere [17] [18] or a high explosive detonation product [19] which releases across a vacuum gap stagnating on the plate, and different driving techniques and the gap thickness determine the magnitude of the driving pressure and the strain rate of the plate.

Based on the energy balance method of Mises [11], that is, the sum of the rate of kinetic and potential energies and the average stress power integral equals zero, namely

$$
\frac{\partial}{\partial t}(\langle T\rangle+\langle V\rangle)+\int_{0}^{h}\langle\dot{W}\rangle \mathrm{d} y=0
$$

where $\dot{W}=S_{i j} D_{i j}$ is the stress power, $S_{i j}$ is the deviatoric stress tensor, $D_{i j}$ is the deformation rate tensor.

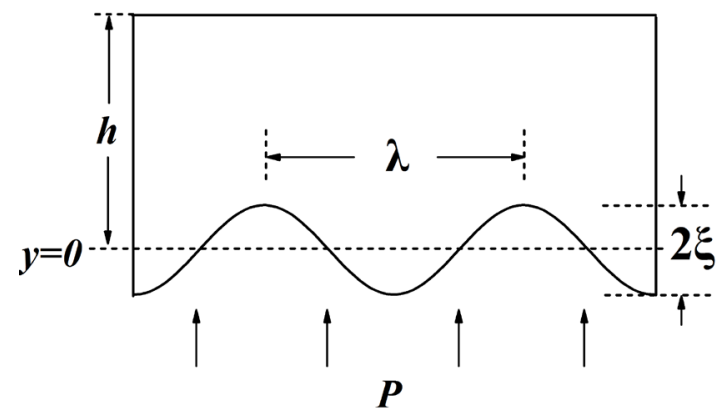

Figure 1. Schematic showing RTI in a finite thickness plate under load. 
The aforementioned analysis shows that it is very important to compute the stress power in the linear analysis. For the incompressible continuum media, the deformation rate tensor is

$$
D_{i j}=\frac{1}{2}\left(\frac{\partial u_{i}}{\partial x_{j}}+\frac{\partial u_{j}}{\partial x_{i}}\right)=k \dot{\xi} \mathrm{e}^{-k y}\left[\begin{array}{cc}
\cos k x & -\sin k x \\
-\sin k x & -\cos k x
\end{array}\right]
$$

Based on the elastic constitutive relation, deviatoric stress tensor follows

$$
\dot{S}_{i j}=2 G D_{i j}
$$

Under the initial condition $\xi(t=0)=\xi_{0}$, the deviatoric stress tensor is

$$
S_{i j}=2 G k\left(\xi-\xi_{0}\right) \mathrm{e}^{-k y}\left[\begin{array}{cc}
\cos k x & -\sin k x \\
-\sin k x & -\cos k x
\end{array}\right]
$$

So, the stress power in the elastic range is

$$
\dot{W}=S_{i j} D_{i j}=4 G k^{2}\left(\xi-\xi_{0}\right) \dot{\xi} \mathrm{e}^{-2 k y}
$$

While the material deformation is beyond the elastic range, the stress state can be calculated with radial return algorithm [20]. Firstly, the equivalent stress is

$$
s^{*}=\sqrt{3 S_{i j} S_{i j} / 2}=2 \sqrt{3} G k\left(\xi-\xi_{0}\right) \mathrm{e}^{-k y}
$$

Here, we take the equivalent stress at $y=0$ as the effective yield stress $\sigma^{\text {eff }}$, namely

$$
\sigma^{\mathrm{eff}}=s^{*}(y=0)=2 \sqrt{3} G k\left(\xi-\xi_{0}\right)
$$

If $\sigma^{\text {eff }}<\bar{\sigma}$, the deformation is purely elastic,

$$
S_{i j}=S_{i j}
$$

If $\sigma^{\text {eff }} \geq \bar{\sigma}$, the deformation is elastic-plastic, the deviatoric stress tensor $s_{i j}$ is

$$
S_{i j}=\bar{\sigma} / \sigma^{\text {eff }} S_{i j}
$$

$\bar{\sigma}$ is the material yield stress.

Using these results, the stress power integration is obtained,

$$
\int_{0}^{h} \dot{W} \mathrm{~d} y= \begin{cases}2 G k\left(\xi-\xi_{0}\right) \dot{\xi}\left(1-\mathrm{e}^{-2 k h}\right), & \sigma^{\mathrm{eff}}<\bar{\sigma} \\ \bar{\sigma} / \sigma^{\mathrm{eff}} 2 G k\left(\xi-\xi_{0}\right) \dot{\xi}\left(1-\mathrm{e}^{-2 k h}\right), & \sigma^{\mathrm{eff}} \geq \bar{\sigma}\end{cases}
$$

Taking the average kinetic and potential energies and the stress power integration into the energy balance Equation (4), and assuming that $\dot{\xi} \neq 0$, the following evolution equation of the perturbation amplitude of the RTI is obtained:

$$
\ddot{\xi}-g k \xi= \begin{cases}-4 G k^{2}\left(\xi-\xi_{0}\right) / \rho, & \sigma^{\text {eff }}<\bar{\sigma}, \\ -\bar{\sigma} / \sigma^{\text {eff }} 4 G k^{2}\left(\xi-\xi_{0}\right) / \rho, & \sigma^{\text {eff }} \geq \bar{\sigma},\end{cases}
$$

where $\xi(t)$ is the perturbation amplitude on the interface, $\xi_{0}$ is the initial perturbation amplitude, $k=2 \pi / \lambda$ is the wave number, $\rho$ is the material density, $G$ is the material shear modulus, $g=P / \rho h$ is the plate acceleration, $P$ is the 
driving pressure, and $h$ is the plate thickness. When the perfect plastic constitutive model is used, the analytic solution is obtained from (14). Here, the JC and SG constitutive models are applied. The JC constitutive model [21], in which strain-rate and temperature effects are considered, expresses the yield stress in terms of strain, temperature, and strain rate, namely,

$$
\sigma_{\mathrm{JC}}=\left[A+B\left(\varepsilon^{p}\right)^{n}\right]\left[1+C \ln \frac{\dot{\varepsilon}}{\dot{\varepsilon}_{0}}\right]\left[1-\left(\frac{T-T_{r}}{T_{m}-T_{r}}\right)^{m}\right],
$$

where $\sigma_{\mathrm{JC}}$ is the yield stress of the JC model, $A$ is the initial yield stress at a reference temperature, $B$ and $n$ are the material strain hardening parameters, $\varepsilon^{p}$ is the plastic strain, $C$ and $\dot{\varepsilon}_{0}$ are the strain-rate hardening parameter and the defect density characteristic parameter, respectively, $m$ is the heat softening parameter, $T_{r}$ and $T_{m}$ are the reference temperature and the melting temperature, respectively, and commonly $T_{r}=300 \mathrm{~K}$. Because density and pressure effects are not considered in the JC model, the model is only applicable to the low-pressure region. Moreover, the yield stress is simply linearly related to the logarithmic strain rate in the JC model, so the model cannot characterize the transition from a dislocation slide to a dislocation drag mechanism of distortion, restricting the applicable validity of the model to the condition in which strain rate $<10^{4} \mathrm{~s}^{-1}$. The pressure, temperature, and strain-rate terms are added into the elastic-plastic constitutive equation of the SG model [22], and, moreover, the couplings of pressure and strain rate with the yield stress can be split. Because the yield stress in the SG model depends on the pressure, the constitutive equation and the equation of state are coupled. This coupling relation indicates the pressure hardening feature of metals at high pressure. In the SG model, the shear modulus and the yield stress are

$$
\begin{gathered}
G(P, T)=G_{0}\left[1+\frac{1}{G_{0}}\left(\frac{\partial G}{\partial P}\right)_{0} \eta^{-1 / 3} P+\frac{1}{G_{0}}\left(\frac{\partial G}{\partial T}\right)_{0}(T-300)\right], \\
\sigma_{\mathrm{SG}}=Y_{0}(1+B \varepsilon)^{n}\left[1+A \eta^{-1 / 3} P-\alpha(T-300)\right],
\end{gathered}
$$

respectively, where $Y_{0}$ is the initial yield strength, $G_{0}$ is the initial shear modulus, $(\partial G / \partial P)_{0}$ and $(\partial G / \partial T)_{0}$ are the derivatives of the shear modulus with respect to pressure and temperature at the initial condition, respectively, $A$ and $\alpha$ correspond to $(\partial G / \partial P)_{0} / G_{0}$ and $(\partial G / \partial T)_{0} / G_{0}$, respectively, $B$ and $n$ are strain hardening parameters, and $\eta=\rho / \rho_{0}$ is the compression ratio. The SG model is independent of strain rate formally, but it limits the extent of the strain rate; namely, the strain rate must be $>10^{5} \mathrm{~s}^{-1}$. The reason for this limitation is that, in the SG model, one assumes that the intenerating effect induced by the temperature rise under high-velocity impact counteracts the hardening effect of the strain rate, resulting in a stain-rate-independent constitutive equation. The SG model is the most widely applied high-pressure constitutive model at present, which is capable of characterizing the yield stresses of many metals at high pressures. 


\section{Applications of the Analytical Model}

\subsection{Lorenz's Experiment on Aluminum Using the Omega Laser}

The effect of ultrahigh pressures on the material strength was studied using an Al-6061-T6 plate with a pre-imposed sine perturbation driven by plasma ramp loading on the Omega laser, where the peak pressure was $20 \mathrm{GPa}$ and the average strain rate was $\sim 6 \times 10^{6} \mathrm{~s}^{-1}$. During the course of the experiment, the loading rate is not so fast that the compression wave evolves into a strong shock, so the aluminum sample remains shock-free, with less of a temperature rise. In ref. [17], the peak temperature was assumed to be $\sim 400 \mathrm{~K}$, which is far lower than the melting temperature of Al-6061-T6. In this linear analysis, the initial density of aluminum is $\rho_{0}=2.7 \mathrm{~g} / \mathrm{cm}^{3}$, the compression ratio is 1.17 , the sample thickness is $35.6 \mu \mathrm{m}$, the wavelength of the pre-imposed sine perturbation is 40 $\mu \mathrm{m}$, the amplitude of the perturbation is $3.4 \mu \mathrm{m}$, and the temperature is $400 \mathrm{~K}$. The parameters of the Johnson-Cook and Steinberg-Guinan models of Al-6061-T6 are listed in Table 1 and Table 2.

In contrast to the constant pressure driving in previous work, here, a time-dependent pressure driving term $P(t)=7.5 \times 10^{-3}(t+20)^{4} \mathrm{e}^{-0.2(t+20)}$, where $P$ is in GPa and the unit of $t$ is nanoseconds, is introduced to approximate the experimental driving, as shown in Figure 2. In this computation, three models of the JC, SG, and PP are used to describe the stress-strain relation of the metals under dynamic loading. To study the effect of pressure driving on the RTI in metals, the SG model with time-dependent or constant pressure driving is applied in the linear analysis. Figure 3 presents the perturbation growth factor (namely, the ratio of the current amplitude to the initial amplitude, $\xi / \xi_{0}$ ) and ratio of the yield strength to pressure with the SG model under time-dependent or constant pressure driving, where the constant pressure is the same as the value in ref. [17]; namely, the constant pressure equals the peak value of the time-dependent pressure driving, $P_{c}=P_{\max }(t)$.

Figure 3(a) indicates that the result of the linear analysis under time-dependent loading is comparable to that of the experiment within $10 \%$, but the result under constant loading quickly departs from that of the experiment. When $t<5 \mathrm{~ns}$, the growth factors under constant and time-dependent loading are almost in agreement, which indicates that this time extent falls into the linear

Table 1. Parameters for the JC model of Al-6061-T6.

\begin{tabular}{cccccc}
\hline$A(\mathrm{GPa})$ & $B(\mathrm{GPa})$ & $C$ & $T_{\mathrm{m}}(\mathrm{K})$ & $n$ & $m$ \\
\hline 0.3243 & 0.11385 & $2.0 \times 10^{-3}$ & 950.2 & 0.42 & 1.34 \\
\hline
\end{tabular}

Table 2. Parameters for the SG model of Al-6061-T6.

\begin{tabular}{cccccccc}
\hline $\begin{array}{c}G_{0} \\
(\mathrm{GPa})\end{array}$ & $(\partial G / \partial P)_{0}$ & $\begin{array}{c}(\partial \mathrm{G} / \partial T)_{0} \\
\left(\mathrm{GPa} \cdot \mathrm{K}^{-1}\right)\end{array}$ & $\begin{array}{c}Y_{0} \\
(\mathrm{GPa})\end{array}$ & $B$ & $n$ & $\begin{array}{c}A \\
\left(\mathrm{GPa}^{-1}\right)\end{array}$ & $\begin{array}{c}\alpha \\
\left(\mathrm{kK}^{-1}\right)\end{array}$ \\
\hline 27.6 & 1.7940 & -0.017 & 0.29 & 125.0 & 0.10 & 0.0652 & 0.616 \\
\hline
\end{tabular}




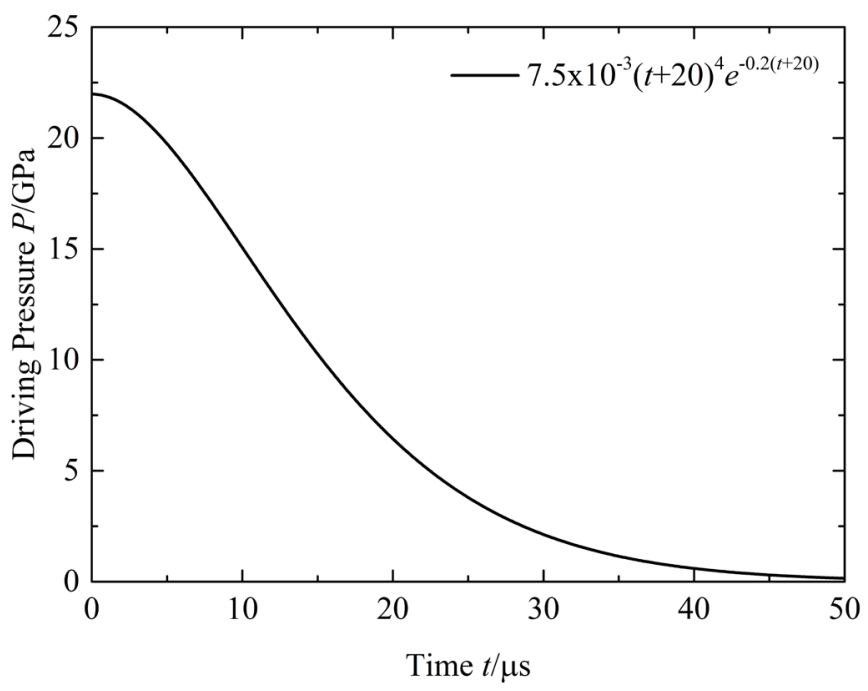

Figure 2. Lorenz's experimental loading history.

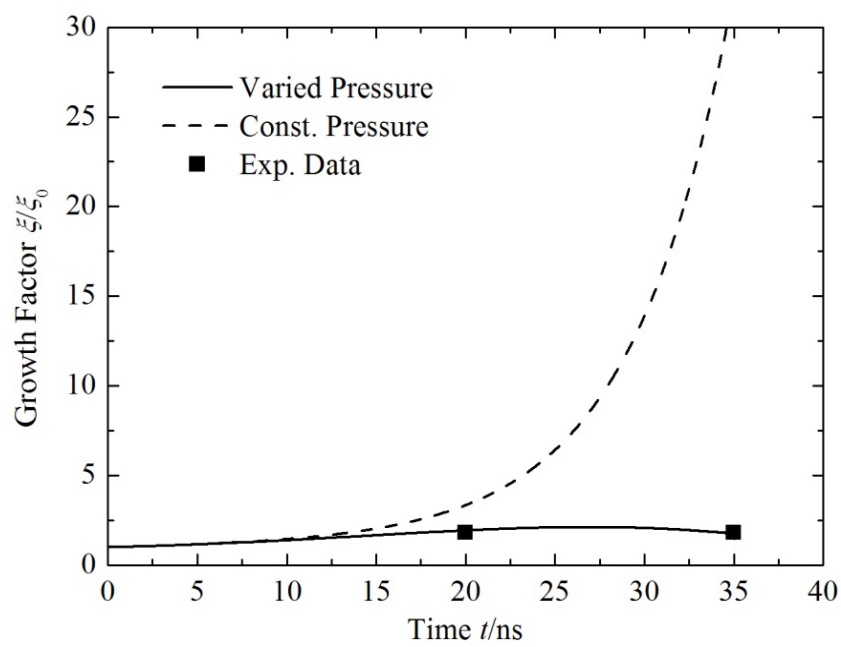

(a)

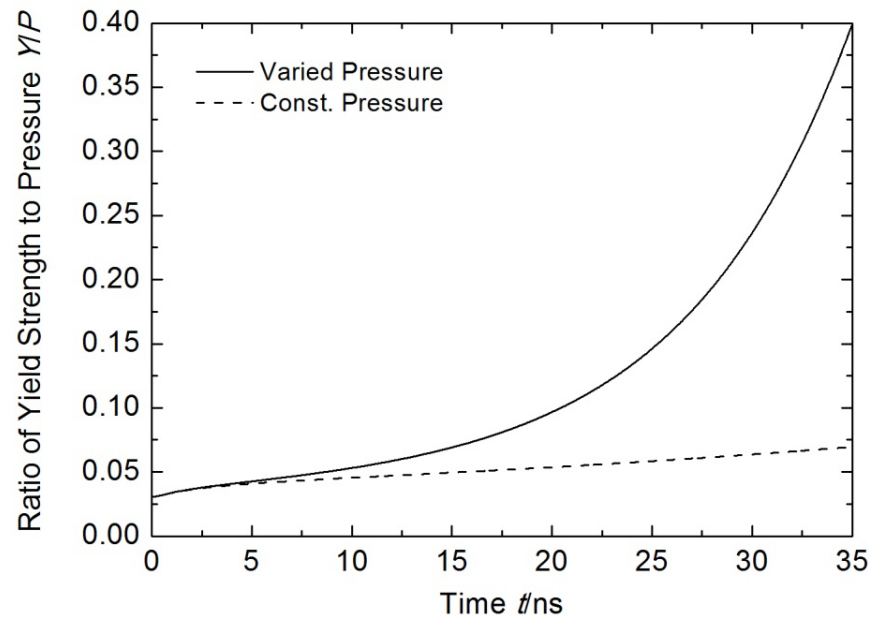

(b)

Figure 3. Growth factor and ratio of yield strength to pressure with the SG model under constant and time-dependent loading. 
region of pressure. In Figure 3(b), when $t>5 \mathrm{~ns}$, the yield strength to pressure $(Y / P)$ value of the time-dependent loading grows rapidly with time, but that of constant loading grows very slowly, which indicates that the yield strength under time-dependent loading inhibits the growth of the RTI in aluminum, but the stability of the yield strength at constant loading is so weak that the growth factor develops rapidly, which differs from the experimental results. As shown, to illustrate the experimental phenomena and obtain results that are in good agreement with experiments, using a time-dependent pressure is indispensable in the linear analysis of the RTI in metals.

The effects of three different constitutive models-namely, the JC, SG, and PP models-on the RTI in metals are further analyzed under time-dependent pressure, as shown in Figure 4. Figure 4(a) presents the comparative results of the

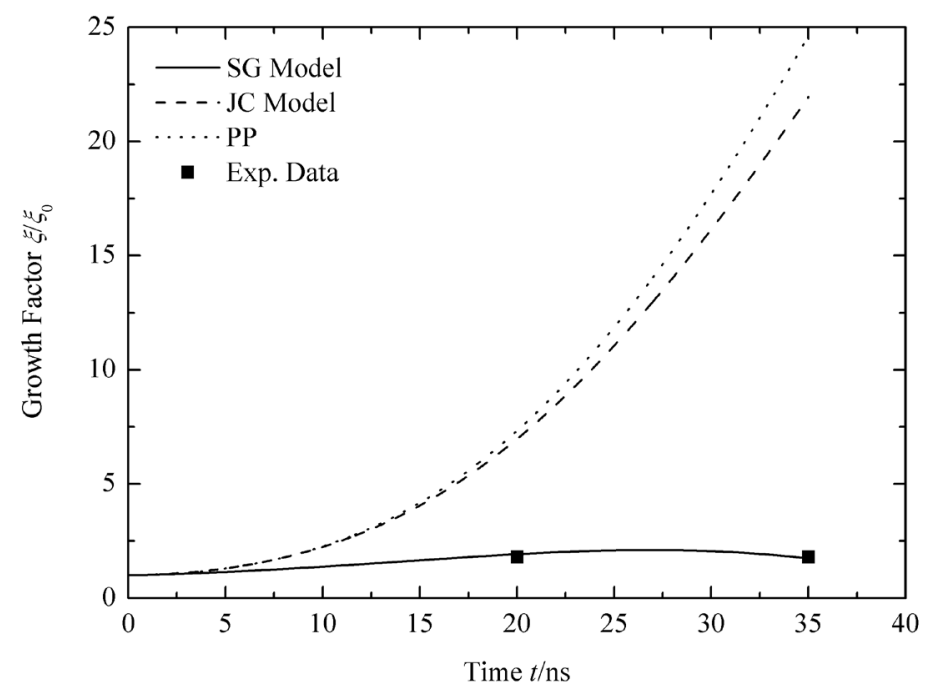

(a)

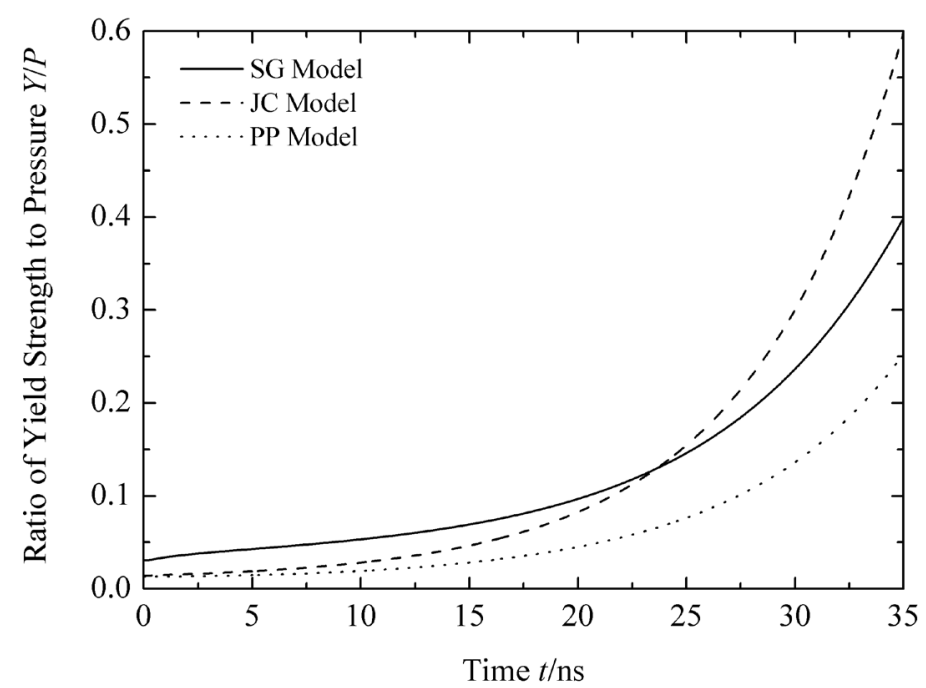

(b)

Figure 4. Growth factor and ratio of yield strength to pressure with the SG, JC, and PP model under time-dependent loading. 
growth factor with the three different constitutive models, which shows that the result with the SG model agrees with the experiment quantitatively but the results with the JC and PP models depart from the experiment. The aforementioned results indicate that the SG model is applicable for the high-pressure, high-strain-rate loading condition with a peak pressure of about $20 \mathrm{GPa}$ and a strain rate of $\sim 10^{6} \mathrm{~s}^{-1}$ in the linear analysis. As shown from Figure 4 (b), the ratios of the yield strength models to the loading pressure for the JC and perfect plasticity are less than that of the SG model, so their stability effects on growth are too weak to induce a departure of the growth factors. This indicates that the JC and PP models are not applicable to the linear analysis of the RTI in metals in a plasma-driven, quasi-isentropic, high-pressure, and high-strain-rate experiment. It can also be seen from Figure 3(b) and Figure 4(a) that, when $t<15 \mathrm{~ns}$, the $Y / P$ values of the PP and JC models are close together and the growth factors are nearly identical, and, when $t>15 \mathrm{~ns}$, the $Y / P$ values depart from each other rapidly, but the growth factors exhibit little discrepancy. These results indicate that the prophase of the quasi-isentropic loading is the dominant regime of the RTI in metals, whereas, in the late regime of the quasi-isentropic loading, due to the rapid decrease of the loading pressure, the drive of the loading pressure becomes weak for the RTI in metals, and the RTI is in a free evolution regime. The linear analysis results of the RTI for the Lorenz experiment are in accordance with the experiment with time-dependent pressure and the SG model for a peak pressure of $\sim 20 \mathrm{GPa}$ and an average strain rate of $\sim 6 \times 10^{6} \mathrm{~s}^{-1}$ in aluminum.

\subsection{Park's Experiment on Vanadium Using the Omega Laser}

The linear analysis method can be applied to Lorenz's experiment with loading pressure and strain rate of the are $\sim 20 \mathrm{GPa}$ and $6 \times 10^{6} \mathrm{~s}^{-1}$. Whether or not the linear analysis is applicable to an experiment with a higher loading pressure and a higher strain rate is not clear. Hence, the linear analysis was performed on the RTI in vanadium, which was quasi-isentropically plasma-driven on the Omega laser by Park et al. [18]. A CH-based epoxy "heat shield" was machined on the front side of the sample to ensure that the surface of the sample is not ablated by the stagnating plasma. The temperature rise of the sample was $\sim 200 \mathrm{~K}$; therefore the temperature of the sample was substantially lower than the melting temperature of vanadium. The peak pressure in the experiment reached $90 \mathrm{GPa}$, the strain rate was $3 \times 10^{7} \mathrm{~s}^{-1}$, the initial density was $\rho_{0}=6.1 \mathrm{~g} / \mathrm{cm}^{3}$, the compression ratio was $\eta=1.3$, the sample thickness was $35 \mu \mathrm{m}$, the wavelength of the imposed ripple was $60 \mu \mathrm{m}$, the amplitude was $0.6 \mu \mathrm{m}$ and the temperature was $T=500 \mathrm{~K}$. The parameters for the Steinberg-Guinan model of vanadium are listed in Table 3.

Because the loading pressure and the strain rate in Park's experiment are substantially beyond the applicable extent of the JC model, here only the SG model is applied in the linear analysis. Both constant and time-dependent pressure 
Table 3. Parameters for the SG constitutive model of vanadium.

\begin{tabular}{cccccccc}
\hline $\begin{array}{c}G_{0} \\
(\mathrm{GPa})\end{array}$ & $(\partial \mathrm{G} / \partial P)_{0}$ & $\begin{array}{c}(\partial \mathrm{G} / \partial T)_{0} \\
\left(\mathrm{GPa} \cdot \mathrm{K}^{-1}\right)\end{array}$ & $\begin{array}{c}Y_{0} \\
(\mathrm{GPa})\end{array}$ & $B$ & $n$ & $\begin{array}{c}A \\
\left(\mathrm{GPa}^{-1}\right)\end{array}$ & $\begin{array}{c}\alpha \\
\left(\mathrm{kK}^{-1}\right)\end{array}$ \\
\hline 48.1 & 0.4906 & -0.0099 & 0.60 & 10.0 & 0.10 & 0.0102 & 0.206 \\
\hline
\end{tabular}

drives are considered, and the time-dependent pressure is $P(t)=14.53(t+10)^{1.4} \mathrm{e}^{-0.14(t+10)}$, where the unit of $t$ is nanoseconds, as shown in Figure 5. The constant pressure equals the peak value of the time-dependent pressure as in [18], namely, $P_{c}=P_{\max }(t)$.

Figure 6 present the growth factors computed with the SG model under constant and time-dependent pressure drives and the ratios of the yield strengths to the loading pressures under two loading conditions, respectively. The linear analysis result with time-dependent pressure is in agreement with the experimental data, which is more applicable than that with constant pressure from Figure 6(a). As shown in Figure 6(b), when $t<10 \mathrm{~ns}$, the $Y / P$ values of the linear analysis are almost equal, and the growth factors under constant and time-dependent pressure drives are also indistinguishable. However, after $10 \mathrm{ns,}$ the $Y / P$ value under the time-dependent pressure drive increases quickly, mitigating the development of the RTI in vanadium, whereas the $Y / P$ value under the constant pressure drive remains almost unchangeable, thus not inhibiting the development of the RTI in vanadium and inducing a quick departure of the growth factor from the experimental data. The linear analysis of the RTI in vanadium by Park et al. indicates that the results using the SG model and a time-dependent pressure drive are quantitatively comparable to the experimental results under the extreme conditions of a peak pressure of $\sim 90 \mathrm{GPa}$ and an average strain rate of $3 \times 10^{7} \mathrm{~s}^{-1}$ in vanadium, and the linear analysis is applicable to the extreme conditions of a peak pressure of $\sim 100 \mathrm{GPa}$ and a strain rate of $10^{7} \mathrm{~s}^{-1}$.

\section{Conclusions}

Based on the energy balance equation, the linear equation of RT interfacial instability for finite thickness plate metal is derived. The constitutive model of materials is extended to JC and SG constitutive models. Using the linear analysis method, we numerically solve the perturbation growth experiment of the RT instability on the Omega aluminum laser and the quasi-isentropic loading of aluminum and vanadium. We have obtained the growth evolution of metal aluminum and vanadium interface perturbation and compared it with the experimental results.

The main results of this paper are as follows: 1) Some nonlinear effects are introduced into the linear analysis by the imported time-dependent pressure and the JC and SG models, and the new method is more applicable to the nonlinear regime of the RTI in metals than the classical method with a constant pressure and an ideal constitutive model. 2) For the RTIs in aluminum and vanadium 


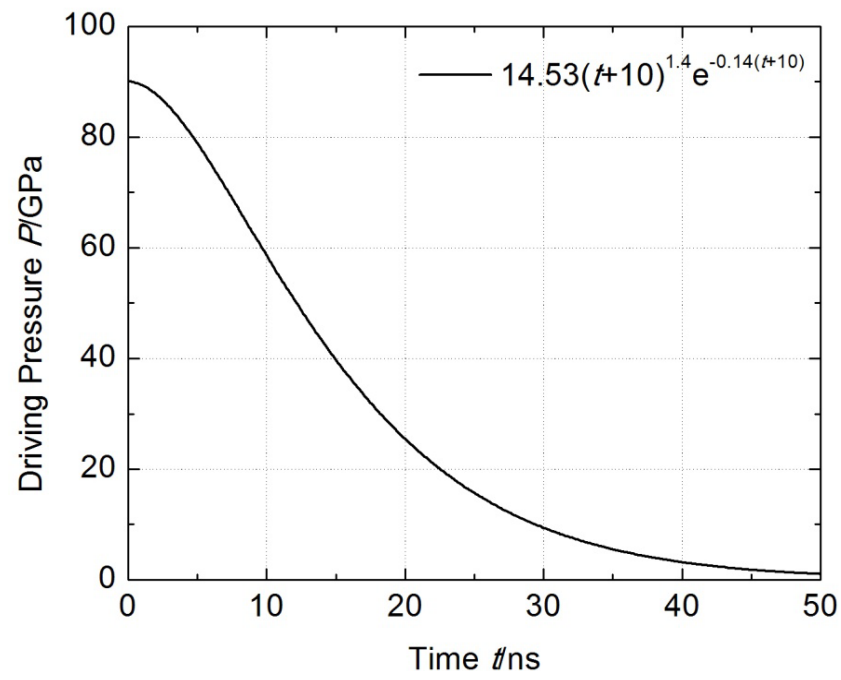

Figure 5. Park's experimental loading history.

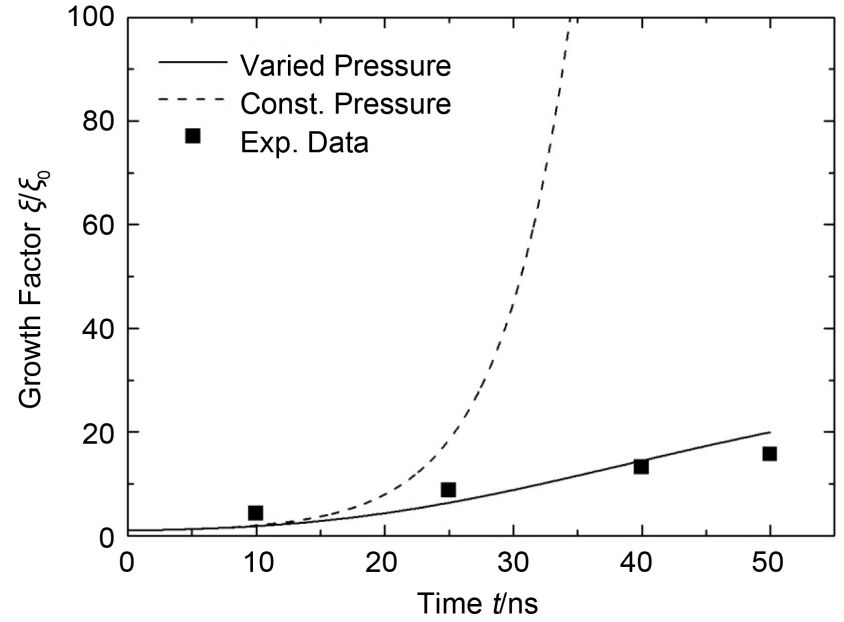

(a)

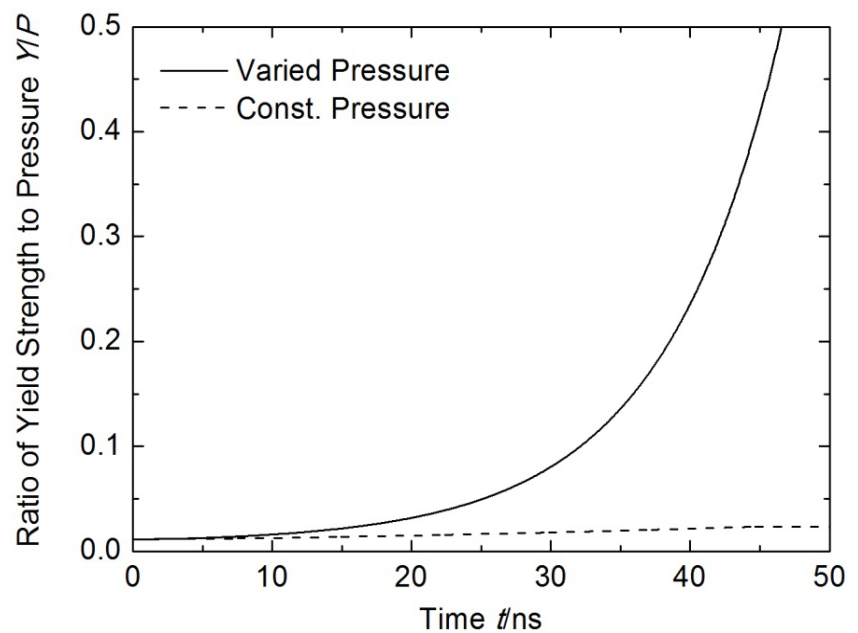

(b)

Figure 6. Growth factor and ratio of yield strength to pressure with the SG model under constant and time-dependent loading. 
quasi-isentropically plasma-driven with high pressures and high strain rates $>$ $10^{5} \mathrm{~s}^{-1}$, the computed results obtained by implementing the SG model in the linear analysis are quantitatively comparable to the experimental data; however, these extreme conditions of high pressures and high strain rates are beyond the applicable extent of the JC model. 3) At the condition of a peak pressure of $\sim 100 \mathrm{GPa}$ and a strain rate of $10^{6}-10^{7} \mathrm{~s}^{-1}$, the linear analysis of the RTI in aluminum and vanadium is also applicable. From the linear analysis of the RTI in metal material, it is found that constitutive models and loading processes are both important, and a better cognitive understanding of the RTI in metals is gained. These results will serve as important references to evolving high-pressure, high-strain-rate experiments and numerical simulations.

\section{Acknowledgements}

This research was supported by the National Natural Science Foundation of China under Grants No. 51572208, and the Joint Fund for Equipment pre Research of the Ministry of Education of China No. 6141A02022209.

\section{References}

[1] Barreras, F., Lozano, A. and Dopazo, C. (2001) Linear Instability Analysis of the Viscous Longitudinal Perturbation on an Air-Blasted Liquid Sheet. Atomization Sprays, 11, 139.

[2] Liang, X., Deng, D.S., Nave, J.-C. and Johnson, S.G. (2011) Linear Stability Analysis of Capillary Instabilities for Concentric Cylindrical Shells. Journal of Fluid Mechanics, 683, 235-262. https://doi.org/10.1017/jfm.2011.260

[3] Liu, Z., Brenn, G. and Durst, F. (1998) Linear Analysis of the Instability of Two-Dimensional Non-Newtonian Liquid Sheets. Journal of Non-Newtonian Fluid Mechanics, 78, 133-166. https://doi.org/10.1016/S0377-0257(98)00060-3

[4] Bai, J.-S., Wang, B., Wang, T. and Liu, K. (2012) Numerical Simulation of the Richtmyer-Meshkov Instability in Initially Nonuniform Flows and Mixing with Reshock. Physical Review E, 86, Article ID: 066319.

https://doi.org/10.1103/PhysRevE.86.066319

[5] Shadloo, M.S., Zainali, A. and Yildiz, M. (2013) Simulation of Single Mode Rayleigh-Taylor Instability by SPH Method. Computational Mechanics, 51, 699-715. https://doi.org/10.1007/s00466-012-0746-2

[6] Dimonte, G. and Schneider, M.B. (1996) Turbulent Rayleigh-Taylor Instability Experiments with Variable Acceleration. Physical Review E, 54, 3740-3743. https://doi.org/10.1103/PhysRevE.54.3740

[7] Grinstein, F.F., Gowardhan, A.A. and Wachtor, A.J. (2011) Simulations of Richtmyer-Meshkov Instabilities in Planar Shock-Tube Experiments. Physics of Fluids, 23, Article ID: 034106. https://doi.org/10.1063/1.3555635

[8] Vandenboomgaerde, M., Souffland, D., Mariani, C., Biamino, L., Jourdan, G. and Houas, L. (2014) An Experimental and Numerical Investigation of the Dependency on the Initial Conditions of the Richtmyer-Meshkov Instability. Physics of Fluids, 26, Article ID: 024109. https://doi.org/10.1063/1.4865836

[9] Prestridge, K., Vorobieff, P., Rightley, P.M. and Benjamin, R.F. (2000) Validation of an Instability Growth Model Using Particle Image Velocimetry Measurements. 
Physical Review Letters, 84, 4353-4356.

https://doi.org/10.1103/PhysRevLett.84.4353

[10] Yoo, J., Mitchell, D., Davidson, D.F. and Hanson, R.K. (2011) Near-Wall Imaging Using Toluene-Based Planar Laser-Induced Fluorescence in Shock Tube Flow. Shock Waves, 21, 523-532. https://doi.org/10.1007/s00193-011-0338-7

[11] Miles, J.W. (1966) General Dynamics Report No. GAMD-7335, AD643161.

[12] Robinson, A.C. and Swegle, J.W. (1989) Acceleration Instability in Elastic-Plastic Solids. II. Analytical Techniques. Journal of Applied Physics, 66, 2859-2872. https://doi.org/10.1063/1.344191

[13] Plohr, B.J. and Sharp, D.H.Z. (1998) Instability of Accelerated Elastic Metal Plates. Zeitschrift für angewandte Mathematik und Physik, 49, 786-804. https://doi.org/10.1007/s000330050121

[14] Terrones, G. (2005) Fastest Growing Linear Rayleigh-Taylor Modes at Solid/Fluid and Solid/Solid Interfaces. Physical Review E, 71, Article ID: 036306. https://doi.org/10.1103/PhysRevE.71.036306

[15] Piriz, A.R., Cela, J.J., Cortazar, O.D., Tahir, N.A. and Hoffmann, D.H. (2005) Rayleigh-Taylor Instability in Elastic Solids. Physical Review E, 72, Article ID: 056313. https://doi.org/10.1103/PhysRevE.72.056313

[16] Piriz, A.R., Cela, J.J. and Tahir, N.A. (2009) Linear Analysis of Incompressible Rayleigh-Taylor Instability in Solids. Physical Review E, 80, Article ID: 046405. https://doi.org/10.1103/PhysRevE.80.046305

[17] Lorenz, K.T., Edwards, M.J., Glendinning, S.G., Jankowski, A.F., McNaney, J., Pollaine, S.M. and Remington, B.A. (2005) Accessing Ultrahigh-Pressure, Quasi-Isentropic States of Matter. Physics of Plasmas, 12, Article ID: 056309. https://doi.org/10.1063/1.1873812

[18] Park, H.-S., Remington, B.A., Becker, R.C., Bernier, J.V., Cavallo, R.M., Lorenz, K.T., Pollaine, S.M., Prisbrey, S.T., Rudd, R.E. and Barton, N.R. (2010) Strong Stabilization of the Rayleigh-Taylor Instability by Material Strength at Megabar Pressures. Physics of Plasmas, 17, Article ID: 056314. https://doi.org/10.1063/1.3363170

[19] Barnes, J.F., Blewett, P.J., McQueen, R.G., Meyer, K.A. and Venable, D. (1974) Taylor Instability in Solids. Journal of Applied Physics, 45, 727-732. https://doi.org/10.1063/1.1663310

[20] Li, Y.C. (2012) Introduction to Tensor Analysis and Modern Continuum Mechanics. University of Science and Technology of China Press, Hefei, 388-390. (In Chinese)

[21] Johnson, G.R. and Cook, W.H. (1983) A Constitutive Model and Data for Metals Subjected to Large Strains, High Strain Rates, and High Temperatures. Proceedings of the 7 th International Symposium on Ballistics, The Hague, 19-21 April 1983, 541-547.

[22] Steinberg, D.J., Cochran, S.G. and Guinan, M.W. (1980) A Constitutive Model for Metals Applicable at High-Strain Rate. Journal of Applied Physics, 51, 1498-1504. https://doi.org/10.1063/1.327799 